\title{
2W Ho:YAG laser intracavity pumped by a diode-pumped Tm:YAG laser
}

\author{
C.Bollig, R.A.Hayward, W.A.Clarkson, and D.C.Hanna
}

\begin{abstract}
Efficient room-temperature operation of a Ho:YAG laser, intracavity pumped by a diode-bar-pumped Tm:YAG laser, is reported. At rod mount temperatures of $10^{\circ} \mathrm{C}$, for both the Tm:YAG and the Ho:YAG rods, we obtained 2.1 W of output at $2.097 \mu \mathrm{m}$ from the Ho:YAG laser for 9.2 $\mathrm{W}$ of diode power incident upon the Tm:YAG rod.
\end{abstract}

Diode-pumped solid-state lasers operating in the eye-safe $2 \mu \mathrm{m}$ spectral region have applications in a number of important areas, including laser radar and medicine. For applications such as laser radar, in addition to the requirement for eyesafe operation, the laser output must be scalable to high (multiwatt) average powers and the operating wavelength must coincide with a spectral region in which there is high atmospheric transmission. For high-power operation in the $\sim 2 \mu \mathrm{m}$ region Tm:YAG is often the preferred choice because of its good thermomechanical properties, which give a high stress-fracture limit, and its convenient pump absorption bands around 782 and $785 \mathrm{~nm}$, which are accessible to commercially available GaAlAs diode bars. Furthermore, Tm:YAG, in common with many other Tm-doped crystals, has the attraction of a pumping quantum efficiency approaching 2 when doped at sufficiently high levels by a cross-relaxation process with neighboring $\mathrm{Tm}^{3+}$ ions. ${ }^{1}$ This process offers the prospect of high lasing efficiencies as well as reduced thermal loading, which are vitally important for power scaling. Recently we demonstrated efficient room-temperature operation of a Tm:YAG laser, end pumped with the reshaped output beam from a 20W diode bar, delivering 4.1 $\mathrm{W}$ of output at $\sim 2 \mu \mathrm{m}$ in a near-diffraction-limited beam for only $13.5 \mathrm{~W}$ of pump power incident upon the Tm:YAG crystal. ${ }^{2}$ Unfortunately, the natural operating wavelength for Tm:YAG (typically $\sim 2.013 \mu \mathrm{m}$ ) lies in a region of the atmospheric transmission spectrum in which there are many absorption lines interspaced by regions of relatively good atmospheric transmission. ${ }^{3}$ Thus, for applications such as laser radar, it is generally necessary to tune the lasing wavelength to a region of high transmission, ${ }^{3}$ thus adding unwanted complexity to the laser system.

Ho:YAG is in this respect an attractive alternative to Tm:YAG, since its lasing wavelength (typically $\sim 2.091$ or $\sim 2.097 \mu \mathrm{m}$ ) coincides with a wavelength region in the atmospheric transmission spectrum with fewer and weaker absorption lines, avoiding the need for wavelength tuning. However, since Ho:YAG has no absorption bands that coincide with the emission wavelengths for commercially available high-power diode bars, it is common practice to codope with $\mathrm{Tm}^{3+}$, which can be pumped by high-power diodes. The $\mathrm{Ho}^{3+}$ is thereby excited by energy transfer from the excited $\mathrm{Tm}^{3+}$ ions. Unfortunately, codoped materials, and particularly YAG, suffer from severe upconversion losses that can result in very significant shortening of the effective lifetime of the upper laser level under typical operating conditions. ${ }^{3}$ One attractive way to avoid the upconversion losses associated with codoped crystals is to use a singly doped crystal of Tm:YAG to intracavity pump a singly doped crystal of Ho:YAG in a common laser cavity. A proof-of-principle demonstration, with Tm:YAG pumped by a Ti:sapphire laser, yielded $120 \mathrm{~mW}$ of output from the Ho:YAG laser at 2.09 $\mu \mathrm{m}$ for $\sim 460 \mathrm{~mW}$ of absorbed pump power ${ }^{4} \mathrm{In}$ practice, for many lidar applications much higher (multiwatt) average powers are generally required. In this Letter we report power scaling by more than an order of magnitude in an efficient Ho:YAG laser producing $\sim 2.1 \mathrm{~W}$ of output at $2.09 \mu \mathrm{m}$ intracavity pumped by a diode-bar end-pumped Tm:YAG laser.

To achieve efficient operation of an intracavitypumped Ho:YAG laser at multiwatt power levels it is first necessary to have a diode-pumped Tm:YAG laser that itself operates efficiently at multiwatt power levels. Scaling Tm:YAG lasers to high average powers while maintaining high efficiency and good beam quality has been a long-standing challenge, owing to their quasi-three-level nature and the relatively low value for $\sigma \tau_{f}(\sim 3$ times smaller than for the 1.064 $\mu \mathrm{m}$ transition in $\mathrm{Nd}$ :YAG). The main problem has been the highly elliptical and inconvenient nature of the output beam from commercially available high-power diode bars, which renders them difficult to focus to the intense circular beams required for efficient end pumping of these low-gain quasi-three-level lasers. To lessen the 
demands placed on pump-beam quality it has been standard practice to cool the Tm:YAG rod to temperatures below $0^{\circ} \mathrm{C}$, and even as low as $-40^{\circ} \mathrm{C}$, to reduce the reabsorption $\operatorname{loss}^{3,5,6}$ However, this approach has the disadvantage of adding considerable further complexity to the laser system. Our solution to this problem is to use a two-mirror beam shaper ${ }^{7}$ to reconfigure the diode-bar output beam with nearly equal $\mathrm{M}^{2}$ values in orthogonal planes so that it can be readily focused by use of a standard arrangement of lenses to a small, nearly circular beam. allowing intense and efficient end pumping. The pump source used in our experiments is a commercially available 20W diode-bar (OPC-AO20-mmm-CS, Opto Power Corporation) temperature tuned to the absorption peak at $\sim 785 \mathrm{~nm}$ in Tm:YAG. The pump-delivery scheme (not shown) was similar to that described in Ref. 7, incorporating a two-mirror beam shaper to equalize the pump beam's $\mathrm{M}^{2}$ values in orthogonal planes and an arrangement of crossed cylindrical lenses to focus the beam to a nearly circular spot with radii $w_{\mathrm{px}} \sim 170 \mu \mathrm{m}$ and $w_{\mathrm{py}} \sim 180 \mu \mathrm{m}$ in orthogonal planes and corresponding $\mathrm{M}^{2}$ values $\mathrm{M}_{\mathrm{x}}{ }^{2} \sim 99$ and $\mathrm{M}_{\mathrm{y}}{ }^{2} \sim 97$, respectively. This choice of focused pump-beam size resulted in a confocal distance in Tm:YAG of $4.4 \mathrm{~mm}$, significantly longer than the absorption length for the pump ( $\sim 1.9 \mathrm{~mm}$ ), thus ensuring a good spatial overlap with the laser mode and hence a low threshold. In our preliminary experiments involving just the Tm:YAG laser, the resonator design used (shown in Fig. 1) was a simple two-mirror cavity consisting of a convex input mirror with high reflectivity $(>99.8 \%)$ in the wavelength range $\sim 2.0$ to $\sim 2.1 \mu \mathrm{m}$ and high transmission $(\sim 90 \%)$ at the diode-pump wavelength, an 8-mm-long Tm:YAG rod with both faces antireflection coated for 2.0-2.1 $\mu \mathrm{m}$ and coated for high transmission $(\sim 93 \%)$ at the diode-pump wavelength, and a concave output coupler with radius of curvature $50 \mathrm{~mm}$ and $3 \%$ transmission at the lasing wavelength $(2.013 \mu \mathrm{m})$. The Tm:YAG rod was doped at a relatively high $\mathrm{Tm}^{3+}$ concentration ( $7 \%$ at.) to ensure efficient two-for-one cross relaxation ${ }^{1}$ to reduce thermal loading and ensure that a large fraction (>98\%) of the pump was absorbed. In our initial work the Tm:YAG rod was mounted in a water-cooled copper heat sink maintained at room temperature $\left(20^{\circ} \mathrm{C}\right)$. We selected the radius of curvature of the convex input mirror as $-150 \mathrm{~mm}$ to provide partial compensation for the thermal lens in the laser rod, which was estimated to have a focal length of 48 $\mathrm{mm}$ at the maximum available pump power. ${ }^{8} \mathrm{With}$ this arrangement, and with a total cavity length of approximately $32 \mathrm{~mm}$, the resonator was stable over the entire range of pump power, with the $\mathrm{TEM}_{\mathrm{oo}}$ beam radius in the Tm:YAG rod calculated to be $127 \mu \mathrm{m}$ at very low pump powers $(<1 \mathrm{~W})$; this value increased slightly with increasing pump power to a beam radius of $130 \mu \mathrm{m}$ at the maximum pump power. For the maximum pump power of $13.5 \mathrm{~W}$ incident upon the Tm:YAG rod, the laser produced $4.1 \mathrm{~W}$ of output at $2.013 \mu \mathrm{m}$ in a near-diffraction-limited beam with $\mathrm{M}^{2}$ beam-propagation factors in orthogonal planes of 1.37 and 1.17 .

For intracavity pumping of Ho:YAG, a similar diode-pumping arrangement was used but with a slightly smaller focused beam radius of $\sim 150 \mu \mathrm{m}$. We modified the cavity design (shown in Fig. 2) to include a $10-\mathrm{mm}$-long Ho-doped ( $0.6 \%$ at.) YAG rod mounted for convenience on the same

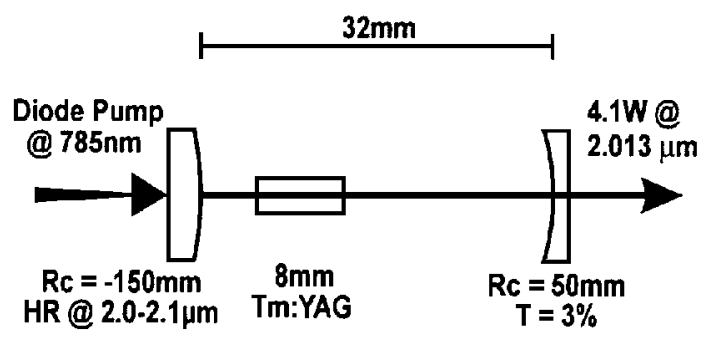

Fig. 1. Single Tm:YAG rod laser resonator design: HR, high reflector; Rc, radius of curvature

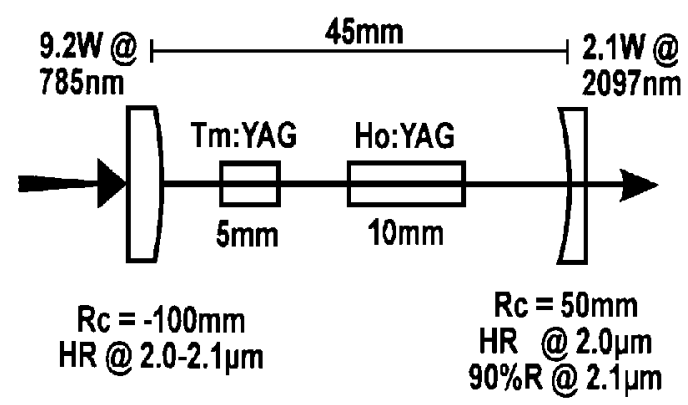

Fig. 2. Intracavity-pumped Ho:YAG laser resonator design. Both rods share a water-cooled heat sink.

water-cooled copper heat sink as the Tm:YAG rod. Both end faces of the Ho:YAG rod were antireflection coated for wavelengths in the range 2.0-2.1 $\mu \mathrm{m}$. In addition, the output coupler was replaced by one with the same radius of curvature but coated for high reflectivity $(>99.9 \%)$ at the Tm:YAG lasing wavelength $(2.01-2.02 \mu \mathrm{m})$ and $\sim 10 \%$ transmission at the Ho:YAG lasing wavelength $\sim 2.1 \mu \mathrm{m}$ ). Owing to a deterioration of 
the antireflection coatings on both faces of the 8-mm-long Tm:YAG, and the lack of an identical replacement rod, a 5mm long Tm:YAG rod was used instead. This rod was also doped with $7 \%$ at.

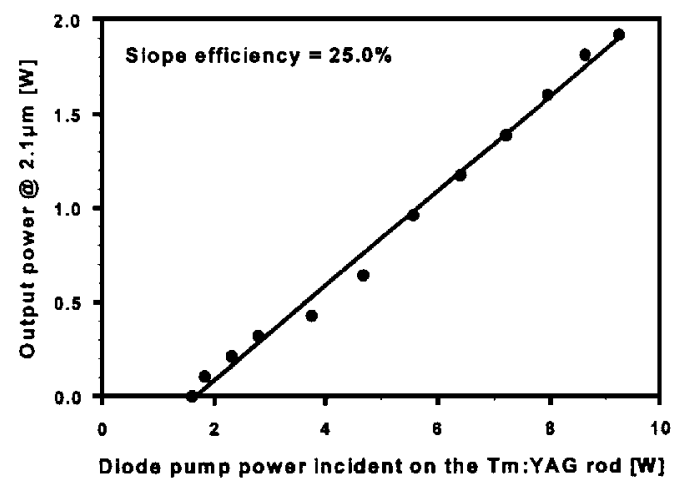

Fig. 3. Output power at $2.1 \mu \mathrm{m}$ versus the diode-bar pump power that was incident upon the rod.

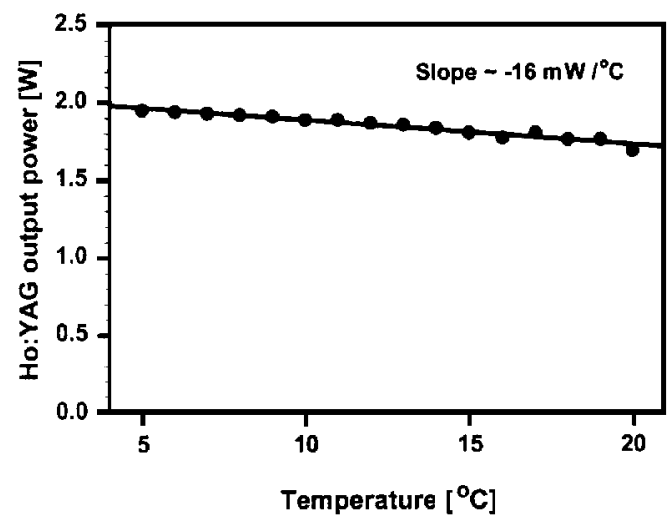

Fig. 4. Output power at $2.1 \mu \mathrm{m}$ versus rod mount temperature.

$\mathrm{Tm}^{3+}$ and gave results for the Tm-only laser comparable with those obtained with the $8 \mathrm{~mm}$ long rod. Owing to the slightly smaller pump-beam size, resulting in slightly stronger thermal lensing in the Tm:YAG rod, in combination with, contribution to the net thermal lensing from heat generated in the Ho:YAG rod, it was necessary to use a convex input mirror of shorter radius of curvature (100 $\mathrm{mm}$ ) to provide the desired degree of compensation of thermal lensing. With this arrangement and a cavity length of $45 \mathrm{~mm}$, the resonator was stable over the full range of available pump power with the calculated $\mathrm{TEM}_{00}$ Tm-laser mode radius in the Tm:YAG rod varying only slightly from $130 \mu \mathrm{m}$ at low pump powers to $135 \mu \mathrm{m}$ at the maximum available pump power. In the latter experiment on intracavity pumping, the maximum available pump power that was incident upon the Tm:YAG rod dropped to $9.2 \mathrm{~W}$, owing to a misalignment of the diode-bar collimating lens and consequent attenuation by the two-mirror beam shaper. Under these operating conditions and at a heat-sink temperature of $10^{\circ} \mathrm{C}$, the maximum output power achieved was $2.1 \mathrm{~W}$ at $2.097 \mu \mathrm{m}$. More typically, the laser operated with a maximum power of $1.9 \mathrm{~W}$ in a beam with $\mathrm{M}^{2}$ beam-propagation factors of 1.6 in the orthogonal planes. Figure 3 shows the output power of the Ho:YAG laser as a function of the diode-pump power that was incident upon the Tm:YAG rod. The slope efficiency of $25 \%$ for the intracavity-pumped Ho:YAG laser is 0.75 times that of the Tm:YAG-only laser indicating very efficient power transfer from the $\sim 2.0 \mu \mathrm{m}$ Tm:YAG output to the $\sim 2.1 \mu \mathrm{m}$ Ho:YAG output. This result is particularly impressive in view of the additional cavity losses owing to the insertion of the Ho:YAG rod.

One interesting feature of this intracavity-pumped Ho:YAG laser is that it exhibited hysteresis behavior close to threshold, where the thresholds for both the Tm and the Ho:YAG lasers (measured in terms of the diode power that was incident upon the Tm:YAG rod) were different depending on whether the pump power was increasing or decreasing. When the diode-pump power was increasing, both lasers reached the threshold for oscillation, almost simultaneously, at a diode power of $2.1 \mathrm{~W}$. However, when the pump power was reduced, the Ho:YAG laser ceased lasing at a significantly lower diode power of $1.7 \mathrm{~W}$, and the Tm:YAG, at an even lower diode power of $1.5 \mathrm{~W}$. This behavior is reminiscent of that of a bistable laser containing a saturable absorber. We believe that the behavior of our laser can be explained by considering the Ho:YAG acting as a saturable absorber for the Tm:YAG laser.

In addition to this hysteresis behavior, we also anticipated the possibility that the Ho:YAG absorption might result in a change in wavelength of the Tm:YAG laser, since it would tend to favor oscillation on wavelengths that reduce its net absorption and hence its cavity loss. This could lead to a decrease in the efficiency of the Ho:YAG laser. Prediction of such behavior is complicated by intensity-dependent bleaching of the $\mathrm{Ho}^{3+}$ absorption. Experimentally we found that the Tm:YAG wavelength $(\sim 2.012 \mu \mathrm{m})$ for the intracavity-pumped Ho:YAG laser was $\sim 1 \mathrm{~nm}$ different from that of the Tm:YAG-only laser.

The temperature dependence of the maximum Ho:YAG output power, plotted in Fig. 4, indicates only a slight reduction in output power with increasing temperature $\left(\sim-16 \mathrm{~mW} /{ }^{\circ} \mathrm{C}\right)$. Thus the 
laser output power was still $>1.7 \mathrm{~W}$, even at room temperature. The absence of a more pronounced decrease in output power with temperature is attributed to the relatively low-threshold pump power for both the Tm:YAG and the Ho:YAG lasers; this is a direct consequence of the relatively intense and therefore efficient pumping afforded by the two-mirror beam-shaping technique.

In conclusion, we have reported efficient high-power operation of a Ho:YAG laser intracavity pumped by a diode-end-pumped Tm:YAG laser, producing as much as $2.1 \mathrm{~W}$ of output at $2.097 \mu \mathrm{m}$ for only $9.2 \mathrm{~W}$ of incident diode power and maintaining efficient operation at room temperature. With optimization of the pump optics to improve the transmission, together with optimum choice of $\mathrm{Tm}^{3+}$ and $\mathrm{Ho}^{3+}$ dopant concentrations and improved resonator design, significant further improvements in efficiency and output power should be readily achieved. The potential for further power scaling with prospects for high-pulse energy Q-switched operation, together with the advantages of its more ideal operating wavelength, make the intracavity-pumped $\mathrm{Ho}^{3+}$ :YAG laser suitable for various laser radar applications.

This work was funded by the Engineering and Physical Sciences Research Council. R.A.Hayward also acknowledges the support of the Defence Evaluation and Research Agency, Portsdown West, in the form of a Cooperative Award in Science and Engineering studentship.

*Present address: Deutsches Zentrum fur Luft-und Raumfahrt, Oberpfaffenhofen, D-82234 Wessling, Germany.

\section{References}

1. T.Becker, R.Clausen, G.Huber, E.W.Duczynski, and P.Mitzscherlich, in Tunable Solid State Lasers, M.L.Shand and H.P.Jenssen, eds., Vol.5 of OSA Proceedings Series (Optical Society of America, Washington, D.C., 1989), p. 150.

2. C.Bollig, W.A.Clarkson, R.A.Hayward, and D.C.Hanna, Opt. Commun. 154,35 (1998).

3. S.W.Henderson, P.J.M.Suni, C.P.Hale, S.M.Hannon, J.R.Magee, D.L.Bruns, and E.H.Yuen, IEEE Trans. Geosci. Remote Sensing 31,4 (1993).

4. R.C.Stoneman and L.Esterowitz, Opt. Lett. 17, 736 (1992).

5. D.C.Shannon, D.L.Vecht, S.Ré, J.Alonis, and R.W.Wallace, Proc. SPIE 1865,136 (1993).

6. W.A.Clarkson and D.C.Hanna, Opt. Lett. 21, 375 (1996).

7. W.A.Clarkson and D.C.Hanna, in Optical Resonators: Science and Engineering, Vol. 45 of NATO ASI Series (Kluwer Academic, Dordrecht, The Netherlands, 1998), p.327.

8. A.E.Siegman, Lasers (University Science, Mill Valley Calif., 1986), p.538. 